\title{
CHARACTERISTICS OF THE UNIQUE MODES IN HBARS
}

\author{
*Shih-Yung Pao ${ }^{1}$, Zuoqing Wang ${ }^{2}$, Zi-Neng Huang ${ }^{2}$, Min-Chiang $\mathrm{Chao}^{2}$, \\ C.S. Lam ${ }^{2}$, and Pei-Zen Chang ${ }^{1}$ \\ ${ }^{1}$ Institute of Applied Mechanics, National Taiwan University, Taipei, Taiwan, R.O.C. \\ ${ }^{2}$ TXC Corporation, No.4, $6{ }^{\text {th }}$ Industry Road, Ping Cheng City, Tao Yuan County, Taiwan, R.O.C
}

\begin{abstract}
Simulation results for three kinds of HBARs with different piezo-film and substrate combinations are presented. Based on the distributions of the resonance frequency spacing and the effective coupling factor $k_{\text {eff }}^{2}(m)$, which significantly rely on the acoustic impedance ratio of the piezo-film to substrate, a unique mode with maximum $k_{e f f}^{2}(m)$ can be chosen. For the unique mode of the HBARs, the variations of the $\mathbf{Q}$-value of the series resonance frequency, $Q_{s}$, the effective coupling factor $k_{e f f}^{2}(m)$, the motional resistance $R_{1}$, the capacitance ratio $r=C_{0} / C_{1}$, the F.O.M. $=Q_{s} C_{1} / 2 C_{0}$, and $Q_{s} \cdot k_{e f f}^{2}(m)$, with the thickness of the substrate are calculated. Those data give some guidelines to choose the thickness of the substrate for compromising the parameters of the operating mode. Results showed that it is unnecessary to choose very thick substrate for obtaining high $Q$. Actually, high $Q$ can be obtained when the thickness ratio to the film reaches about 50 to 150 for the three different kinds of samples.
\end{abstract}

\section{Introduction}

It is well known that a high-stable microwave signal used for frequency control is accomplished by high-Q resonator operating in VHF and UHF. It was reported that the Q-f product exhibited by the types of VHF BAW and UHF SAW resonators often used is on the order of $12 \times 10^{12}$ and $6 \times 10^{12}$, respectively. However, highovertone bulk acoustic resonator (HBAR) consisting of a piezoelectric thin film on a very high-Q substrate, which needs not exhibit piezoelectricity, exhibited a Q-f product more than $10^{14}$. HBAR is an attractive candidate for very high-stability frequency control/selection [1-4].

In an HBAR, there are a few tens to a few hundreds modes appeared in its frequency spectra, for example, for the one in [2], the resonance frequency spacing of the substrate is $2.5 \mathrm{MHz}$, and the fundamental mode of the $\mathrm{ZnO}$ film is $640 \mathrm{MHz}$, so its mode order of the operating mode should be 257 . In application of fixed frequency oscillators, however, only one mode will be used. How to chose the operating mode properly?
High Q-value of the HBAR comes from the extremely high Q-value of the substrate material (one to two orders high than that of the piezo-film), such as spinal, YAG, and boule $[1,4]$, with enough thickness. It was described in [3] that the thicker the substrate the more the energy stored (increase Q-value), but at the same time the narrower the spacing of the resonance frequencies (SRF). But the SRF could not be too small for proper frequency selection applications. Besides, other parameters appeared in the chosen mode are closely related with the thickness of the substrate.

It was analyzed that the SRF is distributed periodically and the value of SFR for some unique modes are given by explicit formulae $[5,6]$. The effective electro-mechanical coupling factor of the unique modes can also be given by explicit formulae [6], and it was shown that the effective coupling factor could be increased by properly choosing the material and thickness of the electrodes [6, 7, 8]. However, there is no quantitative investigation to show the criteria of substrate thickness where the Q-value will reach that of the substrate and there is no quantitative research for the dependence of the HBAR mode parameters on the thickness of the substrate.

In this paper we will emphasize on the criteria of the thickness ratio of the substrate to the piezo-film, where the Q-value reaches to near maximum and other parameters are still comfortable for oscillator applications. In oscillators, the crystal resonator operating in an unique mode of the HBAR is used as a frequency selection device, and its features are indicated by the BVD-equivalent circuit parameters, such as the capacitance ratio $r=C_{0} / C_{1}$, the dynamic resistance $R_{1}$, and the figure of merit (F.O.M) of the mode. Of course, the effective coupling factor $k_{\text {eff }}^{2}(m)$ is an important figure, because it determines the insertion loss of the resonator used in the oscillator. 
We did simulations for comprehensive combinations of a few substrates deposited by different piezoelectric films with different electrodes. For clarity, however, we will only present the results of $\mathrm{ZnO}$-film on three different types of substrates where the acoustic impedance of the substrate is much larger, much smaller and similar with that of the piezo-film. It was found [6,7] that the behaviors of the HBAR crucially depend on the impedance ratio of the two materials. For clearly showing the key points, the effect of the electrode is further ignored in this paper.

In next section, we will present the distributions of the SRF and of the effective coupling factor, $k_{\text {eff }}^{2}(m)$, which are both distributed periodically. Based on their distribution, two clear guidelines for choosing the unique mode will be given. Section III will give the simulation method, the key parameters and the material combinations for the chosen unique modes. Major results for piezo-film $\mathrm{ZnO}$ on three substrates (Quartz, YAG, and a fictitious crystal) are presented in section IV, including the Q-value, $R_{1}$, $k_{\text {eff }}^{2}(N)$, the capacitance ratio, $C_{1} / \mathrm{C}_{0}$, the F.O.M. ( $Q \cdot C_{1} / 2 C_{0}$ ) and $Q \cdot k_{\text {eff }}^{2}(N)$ of the unique mode.

Finally, a summary and a few discussions are given at section V.

\section{Choosing unique mode from frequency spectra of HBAR}

In crystal oscillator circuits, crystal resonator is a twoterminal passive network having highly desirable characteristics those are precisely expressed by its input impedance. Actually, the resonance frequency equations, and by which the SRF and its distribution are obtained from the impedance equation. Furthermore, the effective coupling factors are derived from the electrical input impedance as well.

\section{(1). Input impedance of an HBAR}

For a four-layer HBAR, i.e., a piezoelectric film sandwiched by two electrodes and deposited on a substrate, as shown in Fig.1, the electric input impedance can be given by

$$
Z_{i n}=\frac{V}{I}=\frac{1}{j \omega C_{0}} \cdot\left[1-\frac{k_{t}^{2}}{\gamma} \cdot \frac{\left(z_{1}+z_{2}\right) \cdot \sin \gamma+j \cdot 2(1-\cos \gamma)}{\left(z_{1}+z_{2}\right) \cdot \cos \gamma+j \cdot\left(1+z_{1} z_{2}\right) \cdot \sin \gamma}\right]
$$

where

$$
C_{0}=\varepsilon_{33}^{S} \cdot A / l
$$

is the clamped capacitance of the resonator; $z_{1}=Z_{1} / Z_{0}$ and $z_{2}=Z_{2} / Z_{0}$, and

$$
\begin{gathered}
Z_{1}=j Z_{e 1} \tan \gamma_{e 1} ; \\
Z_{2}=j \frac{Z_{s b} \tan \gamma_{s b}+Z_{e 2} \tan \gamma_{e 2}}{1-\left(Z_{s b} / Z_{e 2}\right) \tan \gamma_{e 2} \tan \gamma_{s b}} ; \\
\gamma=2 \pi f \cdot l / c ; \\
\gamma_{e 1}=\frac{\omega d_{1}}{V_{e 1}} ; \quad \gamma_{e 2}=\frac{\omega d_{2}}{V_{e 2}} ; \quad \gamma_{s b}=\frac{\omega b}{V_{s b}} ; \\
Z_{e 1}=A \cdot \rho_{e 1} \cdot V_{e 1} ; Z_{e 2}=A \cdot \rho_{e 2} \cdot V_{e 2} \\
Z_{s b}=A \cdot \rho_{s b} \cdot V_{s b}
\end{gathered}
$$

$k_{t}^{2}=h_{33}^{2} \cdot \varepsilon_{33}^{S} / C_{33}^{D}$ is the thickness expander mode coupling factor of the piezoelectric film; $z_{b}=Z_{b} / Z_{0}, Z_{\mathrm{b}}$, $z_{1}, z_{2}$ and $Z_{0}$ are the acoustic impedance of the substrate plate, the top and bottom electrode and the film layer, respectively, and $Z_{b}=\rho_{b} \cdot V_{b} \cdot A, Z_{0}=\hat{\rho} \cdot \hat{V} \cdot A, \mathrm{~A}$ is the surface area of the resonator. $\rho_{b}, \rho_{e 1}, \rho_{e 2}, \hat{\rho}$, and $V_{s b}, V_{e 1}, V_{e 2}, \hat{V}$ are the densities and the extensional wave velocities in the $X_{3}$ direction of the substrate, the top and bottom electrode and the film, respectively; $l, d_{1}, d_{2}$ and $b$ are the thickness of the film, the top and bottom electrode and the plate, respectively.

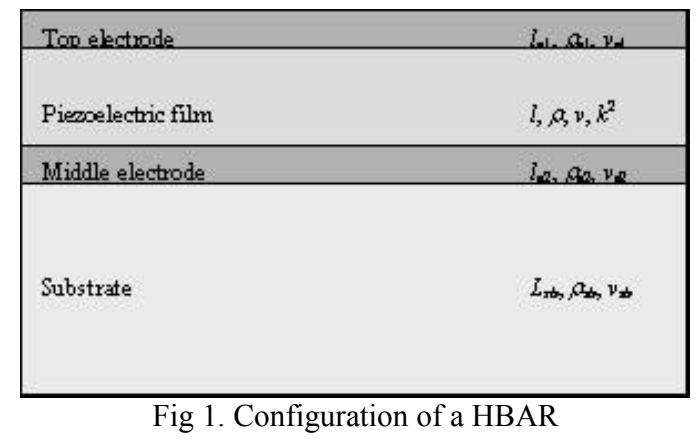

Fig. 2 shows the input electric impedance of a two-layer HBAR (Zno-film on quartz) when ignoring the electrodes. The data for calculating Fig.2 are listed in Table I and the thickness of the film and of the substrate are $4.808 \mu \mathrm{m}$ and $152.87 \mu \mathrm{m}$, respectively. In this sample, the "thickness" of the substrate is $n=(\hat{V} / l) \cdot\left(b / V_{b}\right)=30$, which is the ratio of wave numbers in substrate to that in film.

It is shown in Fig. 2 that there are multiple modes in an HBAR. The larger the $\mathrm{n}$, the more the modes there are. It is our goal to find the unique mode that has the maximum effective coupling factor. Actually, the values of the 
effective coupling factor of all the modes in HBAR are very small. The thicker the substrate, the smaller the effective coupling factor is. For choosing a proper mode operated in a crystal oscillator with reasonable insertion loss, we have to choose the mode with maximum effective coupling factor.

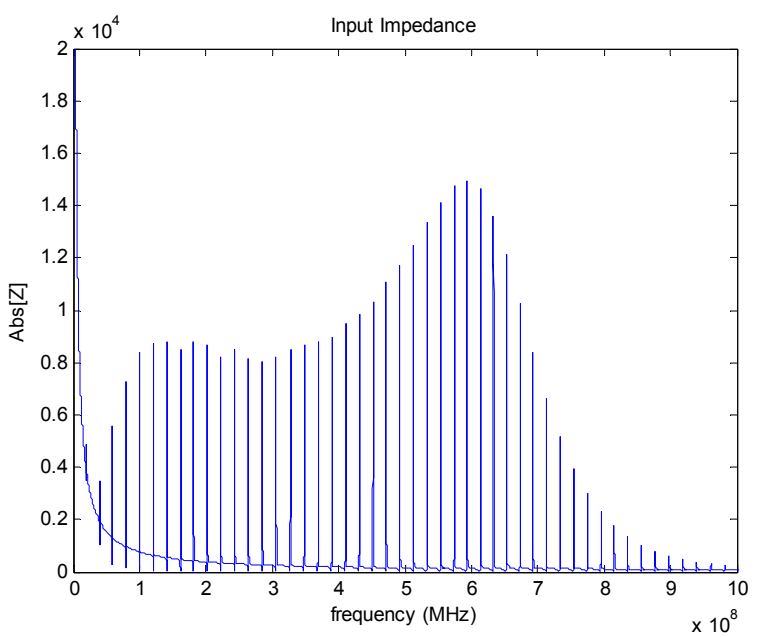

Fig.2 The impedance magnitude of a HBAR

(2). Resonance frequencies and the spacing of the resonance modes

Based on the definition of the IEEE standard [9], the parallel resonant frequency of a single piezoelectric plate corresponds to the maximum resistance, which is the real part of the impedance of the resonator. By using the same definition, the parallel resonant frequencies of the HBAR can be calculated for all the modes. When the materials are loss-less, it is easy to find that the parallel resonant frequency equation is given by

$$
\tan \gamma+z_{b} \tan \gamma_{b}=0
$$

The series resonant frequencies correspond to the maximums of the conductance, which is the real part of $Y_{\text {in }}=1 / Z_{\text {in }}$. They are the roots of the following equation for loss-less materials

$\tan \gamma+z_{b} \tan \gamma_{b}=\frac{k_{t}^{2}}{\gamma}\left[2 \tan (\gamma / 2)+z_{b} \tan \gamma_{b}\right] \tan \gamma$

After obtaining the resonance frequency data, by experiment or simulation, it is easy to calculate the spacing of the parallel resonant frequencies $(S P R F)$ by defining

$$
\Delta f_{p}(m)=f_{p}(m+1)-f_{p}(m)
$$

Where, $f_{p}(m+1), f_{p}(m)$ are the parallel resonance frequencies of $(\mathrm{m}+1)$-th and $\mathrm{m}$-th orders of the HBAR.

By plotting $\Delta f_{p}(m)$ versus the frequency or the order $m$, a periodic distribution can be found and it is shown in Fig. 3. There are four unique values in the figure: (1) the periodicity, $\Delta f_{C}$; (2) the SPRF value at the center of the regular regions, $\Delta f_{N}$; (3) the SPRF value at the center of the transition regions, $\Delta f_{T}$; (4) the modal frequency spacing of the bare plate, $\Delta f_{0}$. It is shown that the SPRF variation amplitude depends on the ratio of the acoustic impedances of the two materials, but it is a small value anyway.

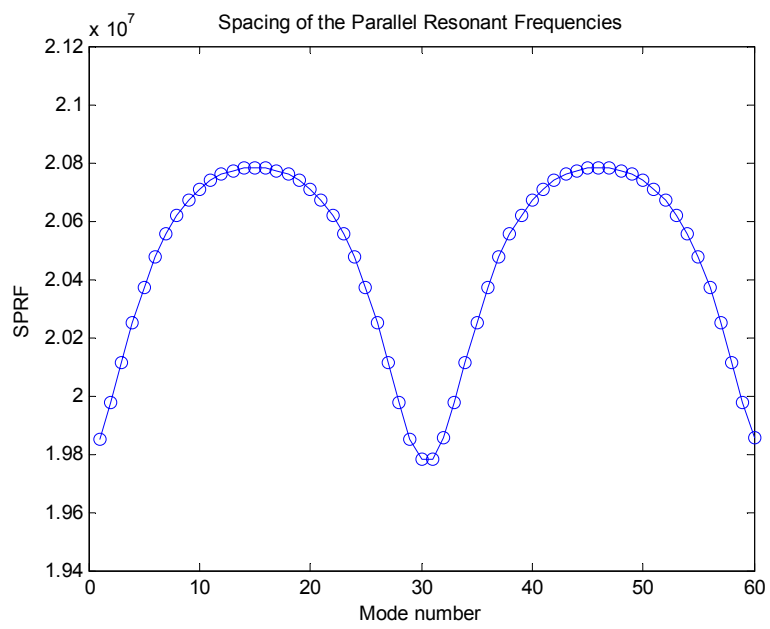

Fig 3. SPRF distribution versus mode order

(a). At the center of the regular regions, where $\gamma \approx n \pi,(\mathrm{n}=0,1,2, \ldots)$, the SPRF value can be derived from (3) and is given approximately by

$$
\Delta f_{N} \approx \Delta f_{0} \cdot\left(1+\frac{2 \cdot(\hat{\rho} l)}{\rho_{b} b}\right)^{\frac{-1}{2}}
$$

Where:

$$
\Delta f_{0}=V_{b} / 2 b
$$

is the mechanical resonance frequency of the fundamental mode, or the spacing of its modes of the bare substrate. By this formula the SPRF of the mode at the center of the regular region can be predicted from the parameters of the substrate and film.

(b). At the center of the transition regions, where $\gamma \approx\left(n+\frac{1}{2}\right) \pi$, the SPRF value can be derived from (3) and given by

$$
\Delta f_{T} \approx \Delta f_{0} \cdot\left(1+\frac{\rho_{b} V_{b}^{2}}{b} \cdot \frac{l}{\hat{C}_{33}}\right)^{-1}
$$


By this formula the SPRF of the mode at the center of the transition region can be predicted from the parameters of the substrate and film. Usually, $l / b<<1$, the factor in (7) and (8) are near 1 and so the spacing of the resonance frequency is basically determined by the ratio of the thickness of the two layers.

(3). Effective coupling factor of the modes in HBAR

In other hand, the definition of effective coupling coefficient $k_{\text {eff }}^{2}(m)$ is

(9)

$$
k_{e f f}^{2}(m)=\frac{\pi^{2}}{4} \cdot \frac{f_{s}(m)}{f_{p}(m)} \cdot\left[1-\frac{f_{s}(m)}{f_{p}(m)}\right]
$$

Where $f_{p}(m), f_{s}(m)$ are the parallel and series resonant frequencies of the $m$-th mode. Plotting $k_{\text {eff }}^{2}(m)$ versus the order $m$, we can obtain a distribution as shown in Fig. 4, which is calculated by the same data of Fig. 2 and 3. It is shown that the variation of $k_{\text {eff }}^{2}(m)$ is significant and the mode with maximum $k_{\text {eff }}^{2}(m)$ is easy to be found.

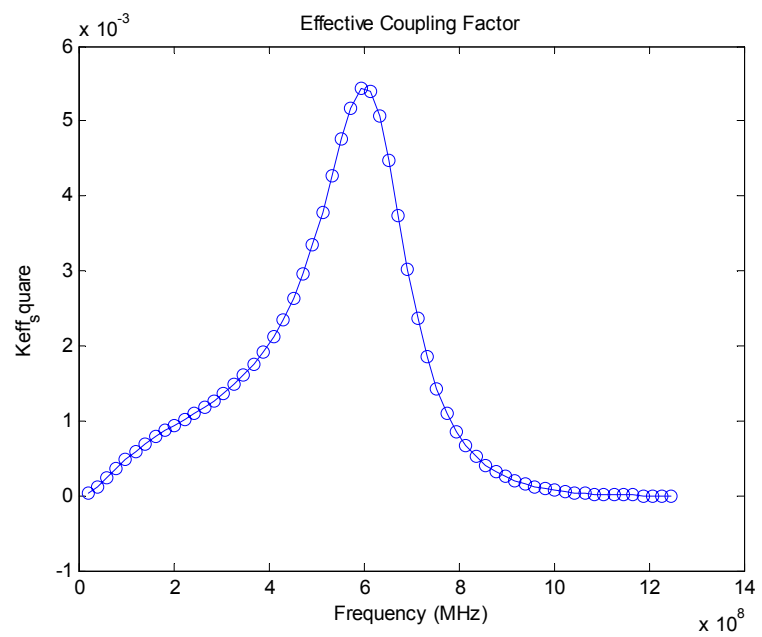

Fig 4. $k_{e f f}^{2}(m)$ distribution versus mode order

Using the definition of the "effective coupling factor" given in Eq.(9) and the resonance frequency equations (3) and (4), we derived two explicit formulae which relate $k_{t}^{2}$ to the "effective coupling factor" for a unique mode at the center of the first normal region, $k_{\text {eff }}^{2}\left(m_{N}\right)$, and at the first transition region, $k_{\text {eff }}^{2}\left(m_{T}\right)$, respectively.
At the first normal region where $\tan (\gamma) \approx 0$, we can write $\gamma=\pi+\varepsilon$ and $\gamma_{b}=m_{N} \pi+\delta$, and ignore the term containing $\varepsilon \cdot \delta$ to obtain the formula

$$
k_{e f f}^{2}\left(m_{N}\right)=\frac{\hat{\rho} l}{\hat{\rho} l+\rho_{s b} b} \cdot k_{t}^{2}
$$

where $m_{N}=\left(\hat{V} b / V_{b} l\right)+1$, and it is the mode order at the center of the region. It will be presented later that we have to choose $(\hat{V} / 2 l) \cdot\left(2 b / V_{s b}\right)$ being an integer for obtain high Q-value.

At the first transition region where $\tan (\gamma) \rightarrow \infty$, we can express $\gamma=(1 / 2) \cdot \pi+\varepsilon$; $\quad$ and $\gamma_{b}=\left(m_{T}+1 / 2\right) \pi+\delta$. After some algebraic manipulation, we obtain the formula

$$
k_{e f f}^{2}\left(m_{T}\right)=\frac{\rho_{s b} \cdot V_{b}^{2} / b}{C_{33}^{D} / l+\rho_{s b} \cdot V_{b}^{2} / b} \cdot k_{t}^{2}
$$

where $m_{T}=\left[\hat{V b} /\left(2 V_{b} l\right)+1 / 2\right]$ and it is the mode order at the center of the transition region. It is noticed that $(\hat{V} / 2 l) \cdot\left(2 b / V_{s b}\right)$ should be an odd integer.

(4). Choosing the unique mode with the maximum effective coupling factor

Simulation results showed that the acoustic impedance ratio of the two layers plays a crucial role for the distribution of the $k_{\text {eff }}^{2}$ versus the mode order. For the case of $z_{b}=Z_{b} / Z_{0}>>1$, the peak value of $k_{\text {eff }}^{2}$ is located at the center of the transition region, which corresponds to a frequency $f=\hat{V} / 4 l$. It seems that the piezofilm is working as a "quarter wavelength resonator". In this case, Eq. (11) can be used to accurately evaluate the effective coupling factor of the unique mode. Contrarily, for the case of $\mathrm{ZnO}$ film on a fused quartz plate resonator, where $z_{b}<<1$, the peak $k_{\text {eff }}^{2}$ value is located near the frequency $f=\hat{V} / 2 l$, and the film acts as a "half wavelength resonator". In this case Eq.(10) can be used to evaluate the effective coupling factor of the unique mode accurately. For the case of YAG where $z_{b}=1.12$, the unique mode with maximum $k_{e f f}^{2}$ is located near the position of $f=(3 / 8) \cdot(\hat{V} / l)$. But Eq. (11) can be used to evaluate the effective coupling factor pretty accurately as well. 
Briefly, the spacing of the modes of HBAR, indicated by the SRF, i.e., $\Delta f_{p}(m)$, varies with the mode order is not significant and basically determined by the thickness ratio of the substrate to film. However, $k_{\text {eff }}^{2}(m)$ of a HBAR varies with mode order significantly and its distribution depends on the impedance ratio of the two materials. When the acoustic impedance of the piezoelectric layer is much smaller than that of the substrate, referred to as a "hard substrate", (there is no such real sample to be quoted), the mode with maximum $k_{e f f}^{2}(m)$ is located in the first transition region. When the acoustic impedance of the piezoelectric layer is much greater than that of the substrate, referred to as a "soft substrate", such as $\mathrm{ZnO}$ on quartz, the maximum magnitude is located in the first normal region. For the case of $\mathrm{ZnO}$ film on YAG, where the acoustic impedance of the two layers are similar, the mode with maximum effective coupling factor is located near the mid-point between the two regions. It is shown that the explicit formulae to evaluate the effective coupling factor are accurate enough.

\section{Q-value and other parameters of the unique mode versus thickness of the substrate}

It is intuitive that the thicker the substrate, the higher the Q-value of the HBAR if the mechanical quality of the substrate is much higher than that of the film (one to two orders higher than that of the piezo-film, such as $\mathrm{ZnO}$-film on spinal, YAG, and boule $[1,4])$. However, when the thickness ratio of the substrate to the film goes to larger, the spacing of the modes will get narrower and other parameters may get worse. Obviously, how to choose the thickness of the substrate for compromising the Q-value and other features is an important topic for crystal oscillator designing.

In crystal oscillator design, the BVD-equivalent circuit parameters are used to describe the performance of the resonance mode. It was indicated $[10,11]$ that if the modes in an HBAR are fully separated, the BVD equivalent circuit model can be used for any given mode. In oscillator applications, the useful parameters of the HBAR are the BVD parameters of the selected unique mode.

The simulation procedure is as follows.

- (1) Choosing one thickness (by using n) and substituting these data into Eq (1), we can find its input impedance distribution and then the data of the series and parallel resonance frequencies, $f_{S}, f_{p}, Q_{S}$, and $k_{\text {eff }}^{2}\left(m_{N}\right)$ or $k_{\text {eff }}^{2}\left(m_{T}\right)$, depending on $z_{b}<1$ or $z_{b}>1$. Here, $Q_{s}=f_{s}(m) / \Delta f_{-3 d B}$, is the Q-value of the series resonance frequency. One can find that for a single-layer resonator, $Q_{s} \rightarrow Q_{m}$.
- (2) The input data are all real except the acoustic velocities of the film and the substrate, i.e., the loss is expressed by a complex velocity, $V=V_{r}+j \cdot V_{i}=V_{r}\left(1+j / 2 Q_{m}\right)$. Here, as usual, the material Q-value, $Q_{m}$, is expressed in the complex elastic constant while the density of the material is real, i.e.,

$$
C=C_{r}+j C_{i}=C_{r}\left(1+j \frac{C_{i}}{C_{r}}\right)=C_{r}\left(1+j / Q_{m}\right)
$$

Where, $Q_{m}=C_{r} / C_{i}$ is the material-Q, and so

$$
V=\sqrt{C / \rho}=\sqrt{C_{r} / \rho} \cdot\left(1+j / 2 Q_{m}\right)=V_{r}\left(1+j \cdot \frac{V_{i}}{V_{r}}\right)
$$

$$
\text { i.e., } V_{r} / V_{i}=2 Q_{m} \text {. }
$$

- (3) From the data of $f_{S}, f_{p}, Q_{S}$, we deduced the parameters, $L_{1}, C_{1}$ and $R_{1}$ by

$\omega_{S}^{2}=1 / C_{1} L_{1} ; \quad \omega_{p}^{2}=\omega_{S}^{2} \cdot\left(1+C_{1} / C_{0}\right) ; \quad Q_{s}=\omega L_{1} / R_{1}=1 / \omega C_{1} R_{1}$ (14)

and the value $C_{0}$ is evaluated from Eq.(2). Based on the four parameters and the $k_{e f f}^{2}(m)$, we can obtain all the features of a unique mode of the HBAR, such as the capacitance ratio $r=C_{0} / C_{1}$, F.O.M. $=Q_{s} C_{1} / 2 C_{0}$, and $Q_{s} \cdot k_{e f f}^{2}(m)$.

- (4) Changing n-value (thickness of the substrate) but taking it always an integer, we obtained the quantitative relationship of the Q-value and other parameters of the unique mode with the thickness of the HBAR. Because only the substrate thickness is changed, it can be found from Eq.(2) that $C_{0}$ is a constant. Besides, the frequency of the unique mode is kept unchanged, while the unique mode number is changed.

Because the $k_{\text {eff }}^{2}(m)$ distribution of the HBAR crucially depends on the acoustic impedance ratio of the substrate to the film, we present three different types of substrates for one piezo-film--ZnO-film: (1) on quartz, where $z_{b}=Z_{b} / Z_{0}=0.495<<1$, (2) on YAG, where $z_{b}=1.12$ which is near 1 , and (3) on a fictitious substrate 
where $z_{b}=2.0 \gg>1$. For clearly showing the effects of substrate thickness on the performance of the HBAR, we ignore the electrode effects. The material data of the combinations are listed in Table I.

\section{Simulation results}

In the simulations, the thickness of the $\mathrm{ZnO}$-film is kept unchanged, $l=4.808 \mu \mathrm{m}$, which corresponds to $f_{c}=624 \mathrm{MHz}$ for a free resonator. Three kinds of samples are listed in Table I. (\#1) ZnO-film on quartz, (\#2) $\mathrm{ZnO}$-film on YAG and (\#3) ZnO-film on a fictitious crystal (X-crystal in table). Thickness of the substrates change from $n=0,1,2, \ldots 150$. As mentioned, the Q-value of a HBAR is increased with the ratio of thickness of the substrate to film, but there is a kind of oscillation phenomenon of the Q-value with the ratio. The positions of the local maximum values correspond to where the $n$-values are integers. It means that the thickness of the substrate has to be chosen carefully in practical HBAR design.

It is found that for samples of \#1, the center frequency of the unique mode is always at $624 \mathrm{MHz}$, no matter what the $\mathrm{n}$ is. But for samples of \#2, the frequency is at about 468 MHz, i.e., $(3 / 8)(\hat{V} / l)$, and for samples of $\# 3$, it is about $312 \mathrm{MHz}$ i.e., $(\hat{V} / 4 l)$.

Some typical simulation results are given in table II, III and IV, and in Fig.5, 6, 7, and 8.

Fig. 5 showed the $Q_{s}$-values versus with n for the three different kinds of samples. It is shown that the curves start from the lines of $Q_{s}=Q_{m}^{(F)}$ where $\mathrm{n}=0$ and gradually go to the asymptotic lines of $Q_{s}=Q_{m}^{(s)}$. For the sample \#1, $Q_{s}$ reaches about $80 \%$ of the $Q_{m}^{(s)}$ when $\mathrm{n}=50$, and $Q_{s}$ reaches $90 \%$ when $\mathrm{n}$ goes to 150 , as shown in Table II. For the samples \#2 and \#3, the value of $f \times Q$ reaches to $0.7 \times 10^{14}$ and $0.4 \times 10^{14}$, respectively, by taking the substrate thickness being $\mathrm{n}=150$.

Fig. 6, Fig.7 and Fig. 8 showed the variation of $C_{1} / C_{0}$, the F.O.M. and $R_{1}$, versus thickness of the substrates, $\mathrm{n}$, for the three kinds of samples, respectively.

It is easy to find that $R_{1}=1 / \omega_{s} C_{1} Q_{s}=\left(1 / 2 \omega_{s} C_{0}\right) \cdot(1 / F . O . M$.$) , and so R_{1}$ and (F.O.M.) are reciprocal for the case where $\omega_{s}$ and $C_{0}$ are unchanged. Here, the variation of $R_{1}$ is a straight line and (F.O.M.) is its reciprocal. This performance is the same as a single plate resonator.

The variation of $R_{1}$ with thickness is important for choosing the thickness. For example, when $\mathrm{n}=50, R_{1}=2$ $\Omega$; When $\mathrm{n}=150, R_{1}=5 \Omega$, and it is 2.5 times increase as for $Q_{s}$ increase from $80 \%$ to $90 \%$. There is no analytical result of the change-rate of $\partial R_{1} / \partial n$, but it is not difficult to find a kind of empirical formulae to describe them. In practice, the data should include the effects of the electrodes and here we only present its trend qualitatively.

The variations of the figures of $C_{0} / C_{1}$ and the figure of $k_{\text {eff }}^{2}\left(m_{N}\right)$ versus the substrate thickness are the same. For a single plate resonator, $C_{1} / C_{0} \approx\left(8 / \pi^{2}\right) \cdot k_{t}^{2}$, and it means that $r=C_{0} / C_{1}$ and $k_{t}^{2}$ are reciprocal, and here we can find the two curves keeping such a relationship as well.

It can be noticed that for sample $\# 1, C_{1} / C_{0}$ is about $0.3 \%$ for $\mathrm{n}=50$. It will have enough pullability to be used in VCXO. But for the case of $\mathrm{n}=150, C_{1} / C_{0}=0.09 \%$, and so, it is very hard to pull the resonator frequency by applied a capacitance.

Fig. 7 showed that if we take F.O.M. $>20$ as a criteria, for the sample \#1, the thickness of the substrate should be $\mathrm{n}<100$; for $\# 2$, thickness of the substrate, $\mathrm{n}$, can reach to 400 , and for $\# 3, \mathrm{n}$ could reach to 300 .

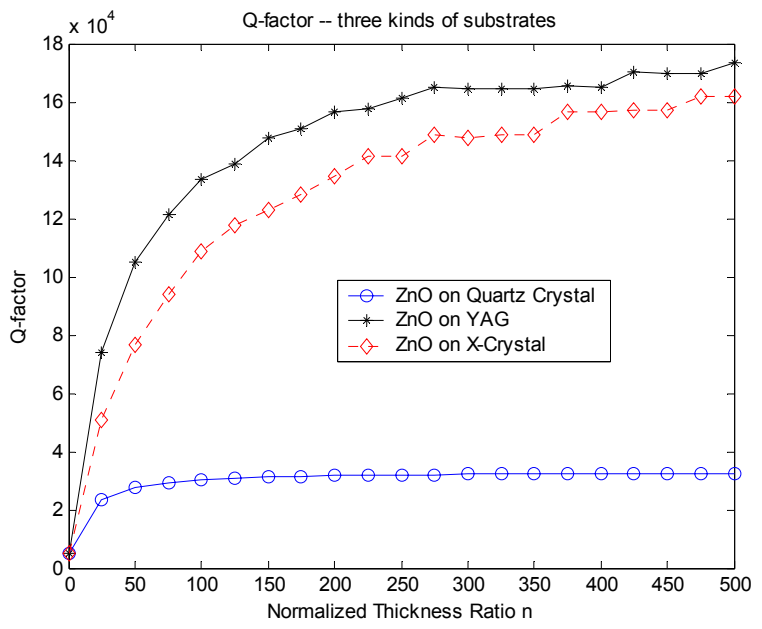

Fig 5. Q-factor for $\mathrm{ZnO}$ on different substrates 


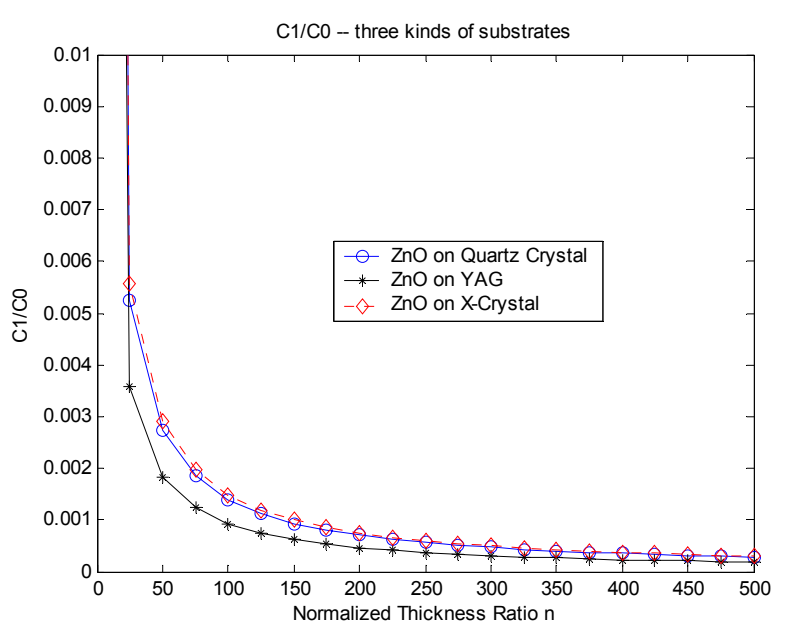

Fig 6. $\mathrm{C} 1 / \mathrm{C} 0$ for $\mathrm{ZnO}$ on different substrates

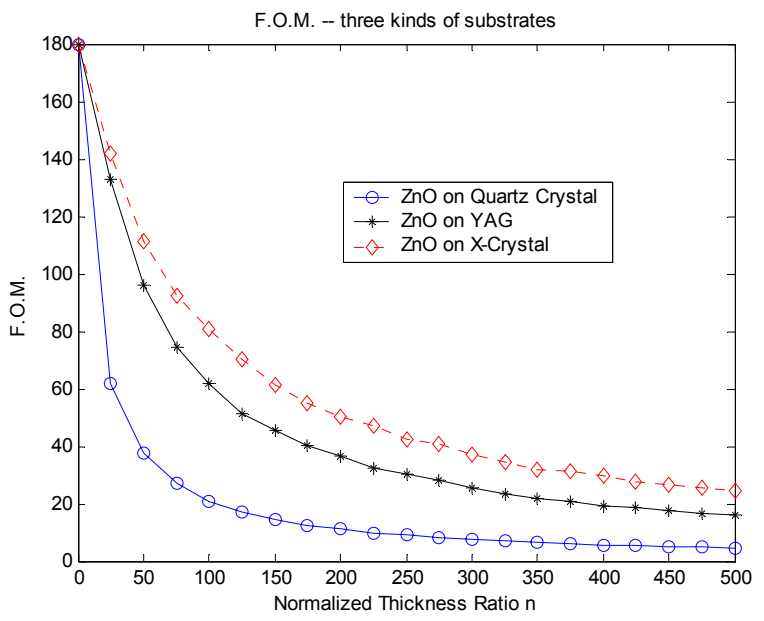

Fig 7. F.O.M. for $\mathrm{ZnO}$ on different substrates

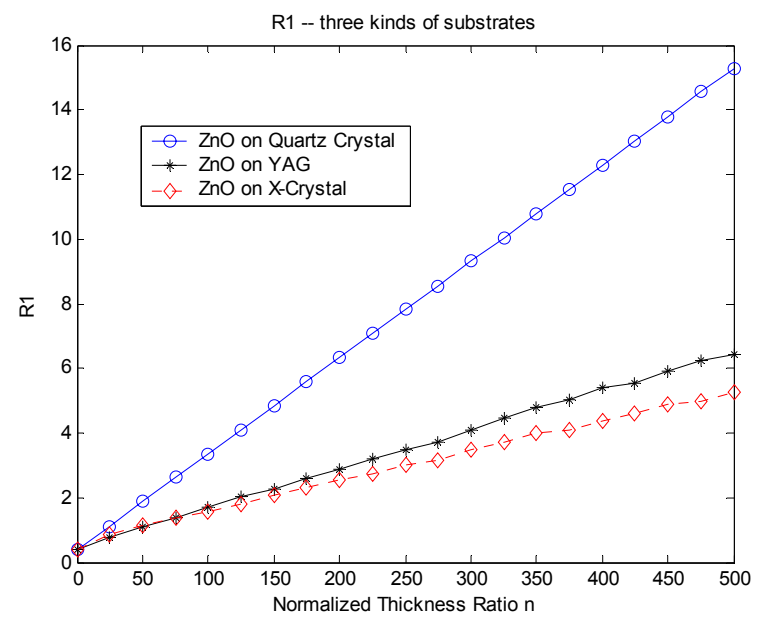

Fig 8. R1. for $\mathrm{ZnO}$ on different substrates

\section{Conclusions and discussions}

In this paper, we presented a set of simulation results for three different piezo-film/substrate combinations. It is indicated that the spacing of the modes of HBAR, indicated by the SRF, i.e., $\Delta f_{p}(m)$, varies with the mode order is not significant and basically determined by the thickness ratio of the substrate to film. However, $k_{\text {eff }}^{2}(m)$ of a HBAR significantly varies with mode order and its distribution depends on the impedance ratio of the two materials. When the acoustic impedance of the piezoelectric layer is much smaller than that of the substrate, i.e., $z_{b} \gg>1$, which is referred to as "hard substrate", (there is no such real sample to be quoted), the mode with maximum $k_{\text {eff }}^{2}(m)$ is located in the first transition region. Contrarily, when the acoustic impedance of the piezoelectric layer is much greater than that of the substrate, i.e., $z_{b}<<1$, referred to as a "soft substrate", such as $\mathrm{ZnO}$ on quartz, the mode with maximum $k_{\text {eff }}^{2}(m)$ is located in the first normal region. For the case of $\mathrm{ZnO}$-film on YAG, where the acoustic impedance of the two layers are similar, the mode with maximum effective coupling factor is located near the mid-point between the two regions.

The results are important for designing a proper HBAR with the unique mode with maximum $k_{e f f}^{2}(m)$. For example, we would like to design an oscillator operating at $624 \mathrm{MHz}$, and using ZnO-film on quartz as shown in sample \#1. The film thickness should be around (less than, because of the effect of electrodes) $4.808 \mu \mathrm{m}$. But if we use $\mathrm{ZnO}$ film on very hard substrate, $z_{b}>>1$, then the film thickness should be a little less than $2.4 \mu \mathrm{m}$, where the center frequency of the free film is at $1,248 \mathrm{MHz}$, and its "quarter wavelength" resonance will be near $624 \mathrm{MHz}$. For the case of $\mathrm{ZnO}$-film on YAG substrate, the $\mathrm{ZnO}$-film should be near $3.6 \mu \mathrm{m}$, and its free resonance frequency is about $832 \mathrm{MHz}$. Then the unique mode frequency of the HBAR will be around its $3 / 8$ wavelength and it is about 624 MHz.

For the unique mode with maximum $k_{\text {eff }}^{2}(m)$, we calculated the variations of the Q-value of the series resonance frequency, $Q_{s}$, the effective coupling factor $k_{\text {eff }}^{2}(m)$, the motional resistance $R_{1}$, the capacitance ratio $r=C_{0} / C_{1}$, the F.O.M. $=Q_{s} C_{1} / 2 C_{0}$, and $Q_{s} \cdot k_{e f f}^{2}(m)$, 
with the thickness of the substrate. It was indicated that most of the relations for the unique mode in HBAR are different with the single-layer resonator. Those data give some guidelines to choose the thickness of the substrate for compromising between the parameters of the operating mode. It seems like that it is unnecessary to choose very thick substrate for obtaining high Q. Actually, for samples with high Q-value substrates such as YAG, $f \times Q=0.7 \times 10^{14}$ can be obtained when the thickness ratio to the film is less than 150 . By using such a thickness, other parameters, such as $k_{\text {eff }}^{2}(m), R_{1}, r=C_{0} / C_{1}$, and the F.O.M. will be much easier to be managed than the case of using large $n$.

* e-mail: sypao@mems.iam.ntu.edu.tw

\section{References:}

[1]. M.M. Driscoll, R.A. Jelen and N. Matthews, "Extremely low phase noise UHF oscillators utilizing high-overtune, Bulk acoustic resonators", Proc. 1990 IEEE Ultrasonics Symposium, 513-518, 1990.

[2]. D.S. Balley, M.M. Driscoll, and R.A. Jelen, "Frequency stability of high-overtune Bulk acoustic resonators", Proc. 1990 IEEE Ultrasonics Symposium, 509-512, 1990.

[3]. J. Rosenbaum, H.L. Salvo, Jr., and S.V. Krishnaswamy, "Overtune response of composite bulk acoustic resonators", Proc. $40^{\text {th }}$ (1986) Annual Frequency Control Symposium, 206-210, 1986.

[4]. H.L. Salvo, Jr. M. Gottlieb and B.R. McAvoy, "Shear mode transducers for high overtone bulk resonators", Proc. $41^{\text {st }}$ Frequency Control Symposium, 388-390, 1987.

[5] Z. Wang, Y. Zhang, and J.D.N. Cheeke,

"Characterization of electromechanical coupling coefficient of piezoelectric film using composite resonators", IEEE Trans. UFFC, Vol. 46 (5), pp. 1327-1330, 1999.

[6]. Y. Zhang, Z. Wang and J.D.N. Cheeke, "Resonant spectrum method to characterize piezoelectric films in composite resonators", IEEE Transactions on Ultrasonics, Ferroelectrics and Frequency Control, Vol.50 (3), pp.321333, 2003.

[7]. M.-C. Chao, Z.-N. Huang, S.-Y. Pao, , Z. Wang and C.S. Lam, "Modified BVD-equivalent circuit of FBAR by taking electrodes into account", 2002 Proc. IEEE

Ultrasonics Symposium, (Munich, Germann, 2002), pp.??????, 2002.

[8]. Shih-Yung Pao, Min-Chiang Chao, Zuoqing Wang, Chih-Hung Chiu, Kung-Chih Lan, Zi-Neng Huang, Lung-
Rung Shih, Chi-Lin Wang, "Analysis and experiment of HBAR frequency spectra and applications to characterize the piezoelectric thin film and to HBAR design", Proc. 2002 IEEE International Frequency Control Symposium, pp. 2002. [9] IEEE Standard on Piezoelectricity, ANSI/IEEE Std 1761987.

[10] F.S. Hickernell, "Measurement techniques for evaluating piezoelectric thin films", Proc. 1996 IEEE Ultrasonics Symposium, 235-242, (San Antonio, 1996).

[11] B.S. Naik, J. J. Lutsky, R. Rief and C. D. Sodini, "Electromechanical coupling constant extraction of thin-film piezoelectric materials using a bulk acoustic wave resonator", IEEE Trans. on UFFC, Vol. 45(1), 257-263, 1998.

\begin{tabular}{|c|c|c|c|c|c|c|}
\hline & $\begin{array}{c}\text { Density } \\
\left(\mathrm{Kg} / \mathrm{m}^{3}\right)\end{array}$ & $\begin{array}{c}Z_{a} \\
\left(10^{6} \mathrm{Kg} /\right. \\
\left.\mathrm{s}^{*} \mathrm{~m}^{2}\right)\end{array}$ & $\begin{array}{c}\hat{V}_{b}, \text { or } \\
(\mathrm{m} / \mathrm{s})\end{array}$ & $\begin{array}{c}k_{t}^{2} \\
(\%)\end{array}$ & $\begin{array}{c}Q_{m} \\
\left(10^{4}\right)\end{array}$ & $\begin{array}{c}Q_{m}^{(s)} / \\
Q_{m}^{(f)}\end{array}$ \\
\hline $\begin{array}{c}\mathrm{ZnO-} \\
\text { film }\end{array}$ & 5,680 & 34.61 & 6,000 & 8.0 & 0.51 & N/A \\
\hline Quartz & 2,651 & 16.85 & 6,359 & N/A & 3.33 & 6.5 \\
\hline YAG & 4,550 & 38.91 & 8,557 & N/A & 18.2 & 35.7 \\
\hline $\begin{array}{c}\text { X- } \\
\text { Crystal* }\end{array}$ & $6,520^{*}$ & $71.72 *$ & $11,007^{*}$ & N/A & $18.2 *$ & $35.7^{*}$ \\
\hline
\end{tabular}

Table I: Data of the materials used in simulations

* A fictitious crystal as substrate

\begin{tabular}{|c|c|c|c|c|c|}
\hline $\mathrm{n}$ & $\begin{array}{c}k_{e f f}^{2}(m) \\
(\%)\end{array}$ & $\begin{array}{c}Q_{s} \\
\left(* 10^{4}\right)\end{array}$ & $\begin{array}{c}C_{1} / C_{0} \\
\left(* 10^{-3}\right)\end{array}$ & F.O.M & $\begin{array}{c}R_{1} \\
(\Omega)\end{array}$ \\
\hline 0 & 7.99 & 0.51 & 70.6 & 180.0 & 0.38 \\
\hline 25 & 6.44 & 2.36 & 5.3 & 62.1 & 1.12 \\
\hline 50 & 3.35 & 2.76 & 2.7 & 37.6 & 1.89 \\
\hline 75 & 2.27 & 2.92 & 1.8 & 26.9 & 2.63 \\
\hline 100 & 1.71 & 3.01 & 1.4 & 21.0 & 3.36 \\
\hline 125 & 1.38 & 3.08 & 1.1 & 17.2 & 4.09 \\
\hline 150 & 1.15 & 3.11 & 0.9 & 14.5 & 4.84 \\
\hline 175 & 0.99 & 3.14 & 0.8 & 12.6 & 5.60 \\
\hline 200 & 0.87 & 3.17 & 0.7 & 11.1 & 6.33 \\
\hline
\end{tabular}

Table II: Typical numerical data for sample of $\mathrm{ZnO}$-film on Quartz substrate 


\begin{tabular}{|c|c|c|c|c|c|}
\hline $\mathrm{n}$ & $\begin{array}{c}k_{e f f}^{2}(m) \\
(\%)\end{array}$ & $\begin{array}{c}Q_{s} \\
\left(* 10^{4}\right)\end{array}$ & $\begin{array}{c}C_{1} / C_{0} \\
\left(* 10^{-3}\right)\end{array}$ & F.O.M & $\begin{array}{c}R_{1} \\
(\Omega)\end{array}$ \\
\hline 0 & 7.99 & 0.51 & 70.6 & 180.0 & 0.38 \\
\hline 25 & 4.41 & 7.40 & 3.6 & 132.9 & 0.77 \\
\hline 50 & 2.25 & 10.50 & 1.8 & 96.2 & 1.08 \\
\hline 75 & 1.51 & 12.15 & 1.2 & 74.7 & 1.39 \\
\hline 100 & 1.14 & 13.32 & 0.9 & 61.6 & 1.69 \\
\hline 125 & 0.92 & 13.85 & 0.7 & 51.4 & 2.03 \\
\hline 150 & 0.76 & 14.74 & 0.6 & 45.6 & 2.28 \\
\hline 175 & 0.66 & 15.06 & 0.5 & 40.0 & 2.60 \\
\hline 200 & 0.57 & 15.63 & 0.5 & 36.4 & 2.89 \\
\hline
\end{tabular}

Table III: Typical numerical data for sample of $\mathrm{ZnO}$-film on YAG substrate 